\title{
Relación entre la gravedad de la infección por Helicobacter pylori y el índice de neutrófilos/ linfocitos y el volumen plaquetario medio en niños Relationship between the severity of Helicobacter pylori infection and neutrophil and lymphocyte ratio and mean platelet volume in children
}

Prof. Asoc. Dr. Yasin Sahin , Dra. Ozlem Gubur y Dra. Emine Tekingunduz

\section{RESUMEN}

Introducción: El Helicobacter pylori (HP) causa inflamación local en el estómago y una respuesta inmunitaria humoral sistémica. En los estudios que investigaron la asociación entre la infección por HPy el índice deneutrófilos/linfocitos(INL) $y$ el volumen plaquetario medio (VPM) en adultos, no se observó ninguna relación. Hasta donde sabemos, no se hicieron estudios en niños. Nuestro objetivo fue evaluar la asociación entre los valores de INL y VPM y la infección por HP, la clasificación de la gravedad y el estado antes y después del tratamiento.

Población y métodos: Enel estudio seincluyeron pacientes de 2 a 18 años de edad con dispepsia y sin enfermedades crónicas sometidos a una endoscopía alta. Sehizo una biopsia endoscópica de la mucosa y se determinaron la presencia de HP y la gravedad de la infección según la clasificación de Sídney.

Resultados: Se incluyeron en el estudio 153 pacientes con HP y 211 sin HP; la media de edad fue de 13,3 $\pm 3,4$ años y 13,1 $\pm 3,5$ años, respectivamente. No se observaron diferencias estadísticamente significativas entre los pacientes con y sin HP ni tampoco entre los subgrupos de pacientes con HP según la gravedad en relación con el INL y el VPM ( $p>0,05)$.

Conclusiones: No se observóuna asociación entre los valores de INL y VPM y la infección por HP, la clasificación de la gravedad ni el estado antes y después del tratamiento.

Palabras clave: niño, endoscopía, Helicobacter pylori, volumen plaquetario medio, indice de neutrófilos/linfocitos.

http:/ / dx.doi.org/10.5546/ aap.2020.e241

Texto completo en inglés:

http:/ / dx.doi.org/10.5546/ aap.2020.eng.e241

Correspondencia:

Dr. Yasin Sahin:

ysahin977@gmail.com

Financiamiento:

Ninguno.

Conflicto de intereses:

Ninguno que declarar.

Recibido: 7-6-2019

Aceptado: 25-11-2019

Cómo citar: Sahin Y, GuburO, TekingunduzE. Relación entre la gravedad de la infección por Helicobacter pylori y el índice de neutrófilos/linfocitos y el volumen plaquetario medio en niños. Arch Argent Pediatr 2020; 118(3):e241-e245.

\section{INTRODUCCIÓN}

El Helicobacter pylori (HP) es una de las causas más frecuentes de infecciones bacterianas crónicas a nivel mundial. Más de la mitad de la población mundial tiene colonización por HP. ${ }^{1}$ Se estimó que la tasa de seroprevalencia del HP era del $33 \%$ en 21 metanálisis de estudios realizados en niños entre 2011 y 2016. Además, se informó que esta tasa sube a aproximadamente el $40 \%$ en los niños sintomáticos. ${ }^{2}$

Es un microorganismo frecuente $(80 \%)$ en los países en vías de desarrollo. El HP causa inflamación crónica en el estómago y provoca una respuesta inmunitaria. ${ }^{3}$ La mayoría de los pacientes suelen ser asintomáticos.

El HP tiende a asentarse en el antro pilórico, que es menos ácido. Además, atrae neutrófilos y linfocitos. Algunas sustancias liberadas por los neutrófilos y las células mononucleares provocan inflamación de la mucosa y, en consecuencia, gastritis. Como resultado, además, se produce una gran infiltración de citocinas, neutrófilos, macrófagos y linfocitos en la mucosa gástrica, que lleva a una inflamación sistémica de bajo grado..$^{3,4}$

El HP es la causa más frecuente de gastritis crónica, y su morbilidad es elevada. Está especialmente asociado con enfermedades gástricas graves, como cáncer de estómago, infección estomacal, úlcera péptica y gastritis

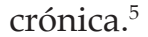

El aumento de los leucocitos en sangre, especialmente los neutrófilos, 
también está asociado con inflamación. ${ }^{6}$ El índice de neutrófilos/linfocitos (INL) se obtiene a partir de un hemograma simple y se usa como marcador inflamatorio de varias enfermedades. Se ha propuesto el uso del INL como factor de predicción independiente en varios problemas clínicos, desde complicaciones cardiovasculares hasta cáncer. ${ }^{7,8}$

Un INL elevado ha demostrado ser un marcador de mal pronóstico en los pacientes sometidos a una intervención cardiovascular y suele ser alto en el síndrome coronario agudo. ${ }^{9}$

Como resultado de los anticuerpos contra el HP que presentan reacción cruzada con las glucoproteínas plaquetarias, se ha sugerido que el recuento de trombocitos disminuye $y$, en consecuencia, se desarrolla trombocitopenia inmunitaria (TPI). En los pacientes con TPI, se observó respuesta positiva en los recuentos de trombocitos tras la erradicación del HP. ${ }^{10,11} \mathrm{En}$ los pacientes con inflamación de bajo grado, como arteriopatía coronaria, y diabetes mellitus, se observaron valores más altos de volumen plaquetario medio (VPM). ${ }^{12}$

Los estudios que investigaron la asociación entre la infección por HP y el INL y el VPM en los adultos son limitados. ${ }^{10,13-15}$ No se observaron diferencias significativas entre los pacientes con y sin HP en relación con el INL y el VPM en los adultos con infección por HP. ${ }^{10,14,15}$ Se ha demostrado que un valor más alto de INL se asoció con la gravedad de la gastritis por HP en los adultos. ${ }^{13}$

En los estudios que investigaron la asociación entre la infección por HP y el INL y el VPM en adultos, no se observó ninguna relación. Hasta donde sabemos, no se hicieron estudios en niños. Nuestro objetivo fue evaluar la asociación entre los valores de INL y VPM y la infección por HP, la clasificación de la gravedad y el estado antes y después del tratamiento.

\section{POBLACIÓN Y MÉTODOS}

Este estudio se llevó a cabo entre marzo de 2017 y octubre de 2018.

\section{Criterios de inclusión}

En el estudio se incluyeron pacientes de 2 a 18 años de edad con dispepsia y sin enfermedades crónicas sometidos a una endoscopía alta.

\section{Criterios de exclusión}

Se excluyó del estudio a los pacientes con cualquier enfermedad orgánica, enfermedad sistémica e infección debido a que cualquier enfermedad concurrente podría alterar los valores de neutrófilos y linfocitos. También se excluyó a los pacientes con metaplasia o atrofia intestinal según la biopsia endoscópica de la mucosa. Y, además, a aquellos que no cumplieron con el tratamiento o que tuvieron fracaso terapéutico.

De los 390 pacientes, se incluyó en el estudio a 364 que tenían síntomas de dispepsia y que recibieron seguimiento en los consultorios de Gastroenterología Pediátrica y se sometieron a una endoscopía alta.

Se obtuvieron, al menos, cuatro muestras para la biopsia en cada paciente, dos del antro pilórico y dos del cuerpo del estómago. Las biopsias se evaluaron según la clasificación de Sídney. ${ }^{16,17}$

Todas las muestras de la biopsia endoscópica de la mucosa se tiñeron con hematoxilina y eosina (HE). Las preparaciones teñidas con HE se evaluaron para determinar su histopatología y la presencia de HP según la clasificación de Sídney. Se detectó a los pacientes con infección por HP y se los incluyó en el grupo de pacientes del estudio. Se los dividió en tres grupos: leve, moderada o grave, según la clasificación de Sídney. A los pacientes con gastritis sin infección por HP se los incluyó en el grupo de referencia. El hemograma se hizo en la sangre periférica antes de la endoscopía. El recuento de leucocitos, neutrófilos, linfocitos y trombocitos se obtuvo a partir del hemograma. El INL se estimó dividiendo el recuento absoluto de neutrófilos por el recuento absoluto de linfocitos. Este fue un estudio retrospectivo, por lo que no se solicitó el consentimiento informado. El Comité de Ética de Mersin Üniversitesi aprobó este estudio (3 de octubre de 2018-385).

Los pacientes recibieron el tratamiento triple de primera línea (inhibidor de la bomba de protones, amoxicilina y metronidazol) durante 14 días según las últimas recomendaciones de la ESPGHAN/NASPGHAN. ${ }^{16}$

El incumplimiento es un factor muy importante en el fracaso terapéutico, por lo que se excluyó del estudio a los pacientes que no cumplieron con el tratamiento. ${ }^{18}$

La eficacia del tratamiento de erradicación del HP se evaluó con una prueba monoclonal de dos pasos para detectar el antígeno del HP en las heces un mes después de finalizar el tratamiento.

\section{Análisis estadístico}

El análisis estadístico se realizó con el programa SPSS, versión 13,0 (SPSS Inc, Chicago, 
IL, EE. UU.). La frecuencia, el porcentaje y la media \pm desviación estándar (DE) se usaron como estadísticas descriptivas. Se utilizó la prueba de la U de Mann-Whitney para comparar los grupos de variables numéricas, mientras que las relaciones entre las variables categóricas se analizaron con la prueba $\chi^{2}$. Las comparaciones entre los grupos se hicieron con el análisis de varianza unifactorial de Kruskal-Wallis para los cinco grupos independientes. Un valor de $p<0,05$ se consideró significativo en términos estadísticos.

\section{RESULTADOS}

Durante el presente estudio, 840 pacientes con dispepsia recibieron seguimiento en los consultorios externos de Gastroenterología Pediátrica. De ellos, 390 se sometieron a una endoscopía alta. De estos 390 pacientes, 17 no cumplieron con los criterios de inclusión: 7 tenían celiaquía; 5 , diabetes mellitus; 3 , gastropatía por antiinflamatorios no esteroides (AINE); 1 , hipotiroidismo; y 1, ataxia-telangiectasia. Después del examen histopatológico, también se excluyó

TABla 1. Características demográficas y de laboratorio de los pacientes y los controles

\begin{tabular}{lccc}
\hline & $\begin{array}{c}\text { Pacientes } \\
(\mathbf{n}=\mathbf{1 5 3})\end{array}$ & $\begin{array}{c}\text { Controles } \\
(\mathbf{n}=\mathbf{2 1 1})\end{array}$ & $p$ \\
\hline Edad (años) $^{+}$ & $13,30 \pm 3,46$ & $13,12 \pm 3,52$ & 0,627 \\
Leucocitos $^{\dagger}$ & $7,47 \pm 2,00$ & $7,67 \pm 2,40$ & 0,390 \\
Hemoglobina (g/dl) $^{+}$ & $12,87 \pm 2,37$ & $12,79 \pm 1,25$ & 0,702 \\
VPM $^{+}$ & $7,87 \pm 0,73$ & $7,87 \pm 0,66$ & 0,995 \\
Recuento de neutrófilos $^{+}$ & $4,24 \pm 1,61$ & $4,39 \pm 2,09$ & 0,452 \\
Recuento de linfocitos $^{+}$ & $2,36 \pm 0,71$ & $2,34 \pm 0,79$ & 0,812 \\
INL $^{\ddagger}$ & $1,76(1,12)$ & $1,76(1,22)$ & 0,093 \\
\hline
\end{tabular}

INL: índice de neutrófilos/linfocitos; VPH: volumen plaquetario medio.

${ }^{+}$Los datos se expresan como media \pm desviación estándar.

${ }^{\ddagger}$ Los datos se expresan como mediana (intervalo intercuartílico).

TABLA 2. Valores antes y después del tratamiento en los pacientes con HP

\begin{tabular}{lccc}
\hline & $\begin{array}{c}\text { Antes del tratamiento } \\
(\mathbf{n}=\mathbf{8 8})\end{array}$ & $\begin{array}{c}\text { Después del tratamiento } \\
\text { (n= 88) }\end{array}$ & $p$ \\
\hline Leucocitos $^{+}$ & $7,74 \pm 2,15$ & $7,16 \pm 2,08$ & 0,013 \\
VPM $^{+}$ & $7,81 \pm 0,72$ & $7,87 \pm 0,79$ & 0,380 \\
Recuento de neutrófilos $^{\dagger}$ & $4,44 \pm 1,71$ & $4,01 \pm 1,90$ & 0,064 \\
Recuento de linfocitos $^{+}$ & $2,40 \pm 0,68$ & $2,32 \pm 0,61$ & 0,195 \\
INL $^{\ddagger}$ & $1,83(1,26)$ & $1,60(0,98)$ & 0,342 \\
\hline
\end{tabular}

INL: índice de neutrófilos/linfocitos; VPH: volumen plaquetario medio.

${ }^{+}$Los datos se expresan como media \pm desviación estándar.

${ }^{\ddagger}$ Los datos se expresan como mediana (intervalo intercuartílico).

TABLA 3. Comparación de los subgrupos con HP según la gravedad de la infección por HP

\begin{tabular}{lccr}
\hline & $\begin{array}{c}\text { Leve } \\
(\mathbf{n}=73)\end{array}$ & $\begin{array}{c}\text { Moderada } \\
(\mathbf{n}=55)\end{array}$ & $\begin{array}{c}\text { Grave } \\
(\mathbf{n}=\mathbf{2 5})\end{array}$ \\
\hline Leucocitos $^{\dagger}$ & $7,21 \pm 2,19$ & $7,69 \pm 1,76$ & $7,73 \pm 1,91$ \\
VPM $^{\dagger}$ & $7,82 \pm 0,69$ & $7,95 \pm 0,83$ & $7,81 \pm 0,64$ \\
Recuento de neutrófilos $^{\dagger}$ & $4,01 \pm 1,75$ & $4,46 \pm 1,35$ & $4,44 \pm 1,69$ \\
Recuento de linfocitos $^{\dagger}$ & $2,35 \pm 0,74$ & $2,35 \pm 0,68$ & $2,42 \pm 0,69$ \\
INL $^{\ddagger}$ & $1,62(1,17)$ & $2,00(0,98)$ & 0,551 \\
\hline
\end{tabular}

INL: índice de neutrófilos/linfocitos; VPH: volumen plaquetario medio.

+ Los datos se expresan como media \pm desviación estándar.

${ }^{\ddagger}$ Los datos se expresan como mediana (intervalo intercuartílico). 
del estudio a 4 pacientes con metaplasia intestinal. A estos pacientes se los excluyó debido a que una enfermedad concurrente y el uso de AINE podrían modificar los valores de neutrófilos y linfocitos. De los 390 pacientes, 364 ingresaron al estudio.

Entre estos, la media de edad de los 153 pacientes en el grupo de pacientes (103 niñas) era de 13,3 $\pm 3,4$ años; mientras que la media de edad de los 211 pacientes en el grupo de referencia (157 niñas) era de 13,1 $\pm 3,5$ años (Tabla 1). No hubo diferencias significativas en relación con la edad y el sexo entre ambos grupos $(p>0,05)$. No se observaron diferencias estadísticamente significativas entre los pacientes con y sin infección por HP en relación con el INL y el VPM $(p>0,05)$ (Tabla 1). Al comparar los valores de INL y VPM de 88 pacientes antes y después del tratamiento, no se observó ninguna diferencia significativa entre ellos $(p>0,05)$ (Tabla 2). No se notaron diferencias estadísticamente significativas entre los subgrupos de pacientes con infección por HP (leve, moderada y grave) en cuanto a los valores de INL y VPM $(p>0,05)$ (Tabla 3$)$.

\section{DISCUSIÓN}

Más de la mitad de la población mundial tiene colonización por HP. Según se informó, aproximadamente, 4,4 mil millones de personas tenían infección por HP en 2015 a nivel mundial. ${ }^{1}$ La prevalencia de HP es alta en los países en vías de desarrollo y, a menudo, se asocia con el nivel socioeconómico y la situación sanitaria. ${ }^{1}$

Los leucocitos y sus subgrupos y el valor del INL demostraron ser indicadores de inflamación sistémica en un estudio previo. ${ }^{19}$

Los estudios que investigaron la asociación entre la infección por HP y el INL y el VPM son limitados. ${ }^{10,13-15}$

Guclu y col. ${ }^{15}$ no hallaron una diferencia significativa entre los pacientes con y sin HP en relación con el INL y el VPM. Los valores de linfocitos y trombocitos estuvieron dentro del intervalo normal en los pacientes con HP, pero fueron significativamente más altos que en aquellos que no tenían HP. El motivo de este aumento se debe probablemente al aumento de la concentración absoluta de linfocitos. Además, la cantidad de casos de gastritis por HP grave fue baja. Los autores sugirieron que se requieren estudios con una mayor cantidad de pacientes con gastritis por HP grave. Al igual que en otro estudio, no se observaron cambios en los valores de VPM en los pacientes con HP. ${ }^{10}$
En un estudio con 50 pacientes con HP y $50 \sin \mathrm{HP}$, los recuentos de leucocitos, linfocitos y neutrófilos fueron más altos en los pacientes con HP en comparación con aquellos sin HP. ${ }^{13}$ También se detectaron valores más altos de INL en los pacientes con HP. Asimismo, los valores más altos de INL se asociaron con la gravedad de la gastritis y una mayor cantidad de síntomas. Los pacientes sin HP presentaron valores de INL significativamente inferiores. Se ha demostrado que estos valores de INL elevados volvieron a la normalidad después del tratamiento y la erradicación con éxito. Según los autores, puede usarse el INL en el seguimiento de los pacientes después de un tratamiento eficaz.

A diferencia de ese estudio, si bien en nuestro estudio incluimos tres veces más pacientes que Farah y col. ${ }^{13}$, no detectamos valores de INL más altos en los pacientes con HP. De manera similar a ese estudio, observamos una disminución del INL después del tratamiento de erradicación del $\mathrm{HP}$, pero no fue estadísticamente significativa. Se requieren estudios adicionales con más pacientes con infección por HP para confirmarlo.

En el estudio de Jakarzadeh y cols., ${ }^{14}$ los valores medios del recuento de leucocitos, del recuento de neutrófilos y del INL fueron significativamente mayores en los pacientes con HP y en el grupo asintomático que en el grupo de referencia. ${ }^{14}$ Los valores medios del recuento de leucocitos, del recuento de neutrófilos y del INL fueron significativamente diferentes entre el grupo asintomático y el grupo con HP. Además, no se notaron diferencias entre los tres grupos en relación con el recuento de linfocitos. Los autores sugieren que los recuentos más altos de leucocitos y neutrófilos en el grupo asintomático se deben probablemente a reacciones microinflamatorias subclínicas causadas por el HP.

En este estudio, no se detectaron diferencias significativas entre los pacientes con y sin infección por HP en relación con el INL y el VPM. No se notaron diferencias estadísticamente significativas entre los subgrupos de pacientes con infección por HP (leve, moderada y grave) en cuanto a los valores de INL y VPM $(p>0,05)$. Al comparar los valores de INL y VPM de 88 pacientes antes y después del tratamiento de erradicación del $\mathrm{HP}$, tampoco se observó ninguna diferencia significativa entre ellos $(p>0,05)$.

Se ha observado una disminución en el recuento de neutrófilos después de la erradicación del HP en un estudio llevado a cabo en Japón. ${ }^{20}$ De manera similar a ese estudio, también detectamos 
una disminución en el recuento de neutrófilos, pero las diferencias no fueron estadísticamente significativas.

Al igual que en dos estudios previos, observamos que los valores de INL y VPM no se correlacionaron con la gravedad de la infección por HP en los niños. ${ }^{10,15}$ Es posible que no detectáramos una relación por el escaso número de pacientes con infección grave por $\mathrm{HP}$ incluidos en este estudio. Por este motivo, se requieren estudios adicionales con más pacientes con infección grave por HP.

\section{Limitaciones}

En primer lugar, este fue un estudio transversal, por lo que no pudimos detectar una relación causal entre el INL, el VPM y el HP. La segunda limitación es la ausencia de un seguimiento a largo plazo después del tratamiento para controlar la respuesta al tratamiento y la falta de control de este marcador con el paso del tiempo. Se midieron el INL y el VPM una sola vez después del tratamiento de erradicación del HP.

A modo de conclusión, no se observó una asociación entre los valores de INL y VPM y la infección por HP, la clasificación de la gravedad ni el estado antes y después del tratamiento.

\section{REFERENCIAS}

1. Hooi JKY, Lai WY, NG WK, Suen MMY, et al. Global prevalence of Helicobacter pylori infection: systematic review and meta-analysis. Gastroenterology. 2017;153(2):420-9.

2. Zabala Torrres B, Lucero Y, Lagomarcino AJ, OrellanaManzano A, et al. Review: prevalence and dynamics of Helicobacter pylori infection during childhood. Helicobacter. 2017;22(5): e12399.

3. Ruggiero P. Helicobacterpyloriand inflammation. Curr Pharm Des. 2010;16(38):4225-36.

4. Kusters JG, van Vliet AHM, Kuipers EJ. Pathogenesis of Helicobacter pylori infection. Clin Microbiol Rev. 2006;19(3):449-90.

5. Kotilea K, Kalach N, Homan M, Bontems P. Helicobacter infection in pediatric patients: Update on diagnosis and eradication strategies. Pediatr Drugs. 2018;20(4):337-51.

6. Nagatomi R. The implication of alterations in leukocyte subset counts on immune function. Exerc Immunol Rev. 2006;12:54-71.

7. Muhmmed Suliman MA, Bahnacy Juma AA, Ali Almadhani AA, Pathare AV, et al. Predictive value of neutrophil to lymphocyte ratio in outcomes of patients with acute coronary syndrome. Arch Med Res. 2010;41(8):618-22.

8. Ubukata H, Motohashi G, Tabuchi T, Nagata H, et al. Evaluations of interferon-gamma / interleukin-4 ratio and neutrophil / lymphocyte ratio as prognostic indicators in gastric cancer patients. J Surg Oncol. 2010;102(7):742-7.

9. Tamhane UU, Aneja S, Montgomery D, Rogers EK, et al. Association between admission neutrophil to lymphocyte ratio and outcomes in patients with acute coronary syndrome. Am J Cardiol. 2008;102(6):653-7.

10. Tan HJ, Goh KL. Extragastrointestinal manifestations of Helicobacter pylori infection: facts or myth? A critical review. J Dig Dis. 2012;13(7):342-9.

11. Arnold DM, Bernotas A, Nazi I, Stasi R, et al. Platelet count response to $H$. Pylori treatment in patients with immune thrombocytopenic purpura with and without $H$. Pylori infection: a systemic review. Haematologica. 2009;94(6):8506.

12. Shah B, Sha D, Xie D, Mohler ER 3rd, Berger JS. The relationship between diabetes, metabolic syndrome, and platelet activity as measured by mean platelet volume: the National Health and Nutrition Examination Survey, 19992004. Diabetes Care. 2012;35(5):1074-8.

13. Farah R, Khamisy-Farah R. Association of neutrophil to lymphocyte ratio with presence and severity of gastritis due to Helicobacter pylori infection. J Clin Lab Anal. 2014; 28(3):219-23.

14. Jakarzadeh A, Akbarpoor V, Nabizadeh M, Nemati M, Rezayati MT. Total leukocyte counts and neutrophillymphocyte count ratios among Helicobacter pylori-infected patients with peptic ulcers: independent of bacterial CagA status. Southeast Asian J Trop Med Public Health. 2013; 44(1):82-8.

15. Guclu M, Faruq Agan A. Association of severity of Helicobacter Pylori infection with peripheral blood neutrophil to lymphocyte ratio and mean platelet volume. Euroasian J Hepatogastroenterol. 2017;7(1):11-6.

16. Jones NL, Koletzko S, Goodman K, Bontemps P, et al. Joint ESPGHAN / NASPGHAN Guidelines for the Management of Helicobacter pylori in Children and Adolescents (Update 2016). J Pediatr Gastroenterol Nutr. 2017;64(6):991-1003.

17. Dixon MF, Genta RM, Yardley JH, Correa P. Classification and grading of gastritis. The updated Sydney System. International Workshop on the Histopathology of Gastritis, Houston 1994. Am J Surg Pathol. 1996;20(10):1161-81.

18. KoletzkoS, Jones NL, Goodman KJ, Gold P, et al. Evidencebased guidelines from ESPGHAN and NASPGHAN for Helicobacter pylori infection in children.J Pediatr Gastroenterol Nutr. 2011;53(2):230-43.

19. Papa A, Emdin M, Passino C, Michelassi C, et al. Predictive value of elevated neutrophil-lymphocyte ratio on cardiac mortality in patients with stable coronary artery disease. Clin Chim Acta. 2008;395(1-2):27-31.

20. Kondo Y, Joh T, Sasaki M, Oshima T, et al. Helicobacter pylori eradication decreases blood neutrophil and monocyte counts. Aliment Pharmacol Ther. 2004;20 Suppl 1:74-9. 